\title{
A Galerkin Method for a Nonlinear Dirichlet Problem
}

\author{
By Jim Douglas, Jr. and Todd Dupont
}

\begin{abstract}
A Galerkin method due to Nitsche for treating the Dirichlet problem for a linear second-order elliptic equation is extended to cover the nonlinear equation $\nabla \cdot(a(x, u) \nabla u)=f$. The asymptotic error estimates are of the same form as in the linear case. Newton's method can be used to solve the nonlinear algebraic equations.
\end{abstract}

1. Introduction. We shall present asymptotic error estimates for a Galerkin method for the approximate solution of the nonlinear Dirichlet problem

$$
\begin{aligned}
& -\nabla \cdot(a(x, u) \nabla u)=f(x) \text { on } \Omega \text {, } \\
& u(x)=g(x) \text { on } \partial \Omega,
\end{aligned}
$$

where $\Omega$ is a bounded domain in $\mathbf{R}^{n}$ with $n \leqslant 3$. Assume that $\Omega$ is locally on one side of its smooth boundary $\partial \Omega$, that $a(x, u)$ is a twice continuously differentiable mapping of $\bar{\Omega} \times \mathbf{R}$ into $\left[\alpha_{0}, \alpha_{1}\right]$, where $0<\alpha_{0} \leqslant \alpha_{1}<\infty$, and that the derivatives of $a(x, r)$ through second order are bounded on $\bar{\Omega} \times \mathbf{R}$. As we shall see later, it follows from well-known regularity results and a uniqueness theorem of Douglas-Dupont-Serrin [1] that if, for some $\alpha \in(0,1), f \in C^{\alpha}(\bar{\Omega})$ and if $g$ can be extended to $\bar{\Omega}$ to be in $C^{2+\alpha}(\bar{\Omega})$, then there exists a unique weak solution $u$ of (1) and, moreover, $u \in C^{2+\alpha}(\bar{\Omega})$. (The class $C^{k+\alpha}(\bar{\Omega})$ consists of all functions whose derivatives through order $k$ are Hölder continuous of order $\alpha$ on $\bar{\Omega}$.)

The Galerkin method we shall employ is the straightforward generalization of a method of Nitsche [3] to this nonlinear case and the form of the asymptotic error estimate produced for this method is essentially the same as in the corresponding linear case. The primary tool used in proving these error estimates is the fact that the formal derivative,

$$
L w=-\nabla \cdot\left(a(x, u) \nabla w+w a_{u}(x, u) \nabla u\right),
$$

of the elliptic operator in (1) is a linear elliptic operator for which the Dirichlet problem has a unique solution; this (nonobvious) fact follows from [1]. The nonsingularity of the Dirichlet problem for (2) is also used in showing that the nonlinear algebraic equations arising in the Galerkin method can be solved by Newton's method provided a sufficiently good approximation of the answer can be found with which to begin the Newton iteration.

2. The Approximate Solution. For $1 \leqslant p \leqslant \infty$ and $k$ a nonnegative integer, let $W_{p}^{k}(\Omega)$ be the functions in $L^{p}(\Omega)$ whose distribution derivatives through order $k$ are also in $L^{p}(\Omega)$. Take \|\|$_{W_{p}^{k}(\Omega)}$ to be the natural norm on $W_{p}^{k}(\Omega)$ :

Received August 7, 1974.

AMS (MOS) subject classifications (1970). Primary 65N30; Secondary 65N15, 65H10, 35J25, $35 \mathrm{~J} 60$. 


$$
\begin{aligned}
\|V\|_{w_{p}^{k}(\Omega)} & =\left[\sum_{|\alpha| \leqslant k}\left\|D^{\alpha} V\right\|_{L^{p}(\Omega)}^{p}\right]^{1 / p}, \quad 1 \leqslant p<\infty, \\
\|V\|_{W_{p}^{\infty}(\Omega)} & =\sup _{|\alpha| \leqslant k}\left\|D^{\alpha} V\right\|_{L^{\infty}(\Omega)}
\end{aligned}
$$

Adopt the following notations:

$$
\|V\|=\|V\|_{L^{2}(\Omega)}, \quad|V|=\|V\|_{L^{2}(\partial \Omega)}, \quad\|V\|_{k}=\|V\|_{w_{2}^{k}(\Omega)} .
$$

Set

$$
\begin{aligned}
H=\left\{v \in W_{6}^{1}(\Omega):\right. & \text { for almost every } x \in \partial \Omega \text { there is an open } \\
& \text { ball } B(x) \text { about } x \text { such that } v \in W_{2}^{2}(\Omega \cap B(x)),
\end{aligned}
$$

$$
\text { and such that } \left.\partial v / \partial v \in L^{2}(\partial \Omega)\right\} \text {, }
$$

where $\partial v / \partial v$ denotes the derivative of $v$ in the direction of the outward normal to $\partial \Omega$. For each $h \in(0,1)$ define a norm on $H$ by

$$
\|\varphi\|^{2}=\|\varphi\|_{h}^{2}=\|\varphi\|_{1}^{2}+h^{-1}|\varphi|^{2}+h|\partial \varphi / \partial \nu|^{2} .
$$

We shall consider a family $\left\{M_{h}\right\}_{0<h \leqslant 1}$ of finite-dimensional subspaces of $H$ satisfying the following assumptions:

(4i) There exists a positive integer $r$ and a constant $c_{1}$ such that, if $2 \leqslant s \leqslant r+1$ and $v \in H^{s}(\Omega)$, then

$$
\inf _{\chi \in v_{h}}\left\{h\|v-\chi\|\left\|_{h}+h^{2}\right\| v-\chi \|_{w_{6}^{1}(\Omega)}\right\} \leqslant c_{1} h^{s}\|v\|_{s} .
$$

(4ii) For each $v \in C_{0}^{\infty}(\Omega)$,

as $h$ tends to zero.

$$
\inf \left\{\|v-\chi\|_{1}: \chi \in M_{h}, \operatorname{supp}(\chi) \subset \subset \Omega\right\}=o(1)
$$

(4iii) For all $\chi \in M_{h}$,

$$
|\partial \chi / \partial \nu| \leqslant c_{1} h^{-1 / 2}\|\chi\|_{1} .
$$

The assumptions (4i)-(4iii) are satisfied by many finite element spaces that contain piecewise polynomials of degree $r$. The condition (4ii) is satisfied by almost all finite element spaces that satisfy (4i) with $o(1)$ replaced by $O\left(h^{r}\right)$; thus, it is a very mild constraint. Condition (4iii) is an inverse hypothesis on the family. $\left\{M_{h}\right\}_{0<h \leqslant 1}$; together with the hypothesis that $\partial \Omega$ is smooth, this condition effectively excludes tensor products of spaces of piecewise polynomials in a single variable. The conditions (4i) and (4iii) are needed for the analysis of Nitsche's procedure even in the case in which the operator is linear, but the need for condition (4ii) is special to the nonlinear problem.

Let $(\because)$ and $\langle\because$,$\rangle denote the scalar products on L^{2}(\Omega)$ and $L^{2}(\partial \Omega)$, respectively. For parameters $\gamma>0$ and $z \in L^{2}(\Omega)$ define a bilinear form $B$ on $H$ by

$$
\begin{aligned}
B(z ; v, w)= & (a(z) \nabla v, \nabla w)-\left\langle a(g) \frac{\partial v}{\partial v}, w\right\rangle \\
& -\left\langle v, a(g) \frac{\partial w}{\partial v}-\gamma h^{-1} w\right\rangle, \quad v, w \in H,
\end{aligned}
$$


where we have suppressed the writing of the $x$ argument of $a$. Note that, if we use $z=v=u$, then $B$ reduces to data in the sense that

$$
B(u ; u, w)=(f, w)-\left\langle g, a(g) \frac{\partial w}{\partial \nu}-\gamma h^{-1} w\right\rangle, \quad w \in H .
$$

The following lemma is an easy consequence of the inverse hypothesis (4iii).

Lemma 1 (Nitsche). There exist positive constants $\rho$ and $\gamma_{0}$, depending only on $\Omega, n, c_{1}, \alpha_{0}$ and $\alpha_{1}$, such that, if $\gamma \geqslant \gamma_{0}$,

$$
\rho\|\| v \|^{2} \leqslant B(z ; v, v), \quad v \in M_{h}, \quad z \in L^{2}(\Omega) .
$$

For each $\gamma>0$ there exists $c_{2}=c_{2}\left(\gamma, \alpha_{1}\right)$ such that

$$
|B(z ; v, w)| \leqslant c_{2}\|v\|\|\||w| \|, \quad v, w \in H, z \in L^{2}(\Omega) .
$$

We shall assume henceforth that $\gamma \geqslant \gamma_{0}$ and is fixed; hence $c_{2}$ is also fixed and (7) holds.

Define an approximate solution $u_{h}$ to be an element of $M_{h}$ satisfying

$$
B\left(u_{h} ; u_{h}, w\right)=(f, w)-\left\langle g, a(g) \frac{\partial w}{\partial \nu}-\gamma h^{-1} w\right\rangle, \quad w \in M_{h} .
$$

To see that there exists at least one solution $u_{h}$ of (9) consider the map $S: M_{h} \rightarrow M_{h}$ defined by

$$
B(y ; S y, w)=(f, w)-\left\langle g, a(g) \frac{\partial w}{\partial \nu}-\gamma h^{-1} w\right\rangle, \quad w \in M_{h}
$$

The condition (7) implies the nonsingularity of the finite set of linear equations that define $S$, and choosing $w=S y$ in (10) shows that the range of $S$ is contained in a ball. Since $S$ is clearly continuous, the Brouwer fixed point theorem implies that (9) has a solution.

3. Convergence Result. Our main result for the procedure given by (9) is the following theorem.

THEOREM 1. Suppose that, for some $\alpha \in(0,1), f \in C^{\alpha}(\bar{\Omega})$ and $g$ can be extended to be in $C^{2+\alpha}(\bar{\Omega})$. Suppose also that $u \in W_{2}^{s}(\Omega)$ for some s satisfying $2 \leqslant s \leqslant$ $r+1$. Then there exists a constant $c_{3}$ such that for $h$ sufficiently small

$$
\left\|u-u_{h}\right\|+h\left\|u-u_{h}\right\| \leqslant c_{3} h^{s} .
$$

Proof. Because of the finite dimensionality of the $M_{h}$ 's the infimum in (4i) is actually assumed; let $\chi \in M_{h}$ be such that $\|u-\chi\| l+h\|u-\chi\|_{W_{6}(\Omega)}$ is minimal. Note that (7), (9) and (5) imply that

$$
\begin{aligned}
\rho\left\|u_{h}-\chi\right\| \|^{2} & \leqslant B\left(u_{h} ; u_{h}-\chi, u_{h}-\chi\right) \\
& =B\left(u ; u-\chi, u_{h}-\chi\right)+\left(\left(a(u)-a\left(u_{h}\right)\right) \nabla \chi, \nabla\left(u_{h}-\chi\right)\right) \\
& \leqslant\left(c_{2}\|u-\chi\| 1+\left\|a(u)-a\left(u_{h}\right)\right\|_{L^{3}(\Omega)}\|\chi\|_{w_{6}^{1}(\Omega)}\right)\left\|u_{h}-\chi\right\| .
\end{aligned}
$$

Thus, by (4i) and the choice of $\chi$,

$$
\left\|u_{h}-u\right\| \leqslant C\left[h^{s-1}+\left\|a(u)-a\left(u_{h}\right)\right\|_{L^{3}(\Omega)}\right] .
$$


Using the fact that $a$ is uniformly Lipschitz continuous with respect to $u$ and the interpolation result (since $\operatorname{dim}(\Omega) \leqslant 3$ )

we see that

$$
\|\varphi\|_{L^{3}(\Omega)} \leqslant C\|\varphi\|^{1 / 2}\|\varphi\|_{1}^{1 / 2},
$$

$$
\left\|u_{h}-u\right\| \leqslant C\left[h^{s-1}+\left\|u-u_{h}\right\|\right] .
$$

In order to bound $\left\|u-u_{n}\right\|$ we use a duality argument. Note that it follows directly from well-known elliptic regularity and Theorem 2 of [1] that any Dirichlet problem for the linear elliptic operator $L$ defined in (2) has at most one weak solution in $W_{2}^{1}(\Omega)$. It then follows that for any $\zeta \in L^{2}(\Omega)$ there exists a unique $\varphi \in W_{2}^{2}(\Omega)$ such that

$$
L^{*} \varphi=\zeta \quad \text { on } \Omega, \quad \varphi=0 \text { on } \partial \Omega,
$$

where $L^{*}$ is the formal adjoint of $L$ :

$$
L^{*} w=-\nabla \cdot(a(x, u) \nabla w)+a_{u}(x, u) \nabla u \cdot \nabla w .
$$

Also there exists a constant $C$ independent of $\zeta$ such that

$$
\|\varphi\|_{2} \leqslant C\|\zeta\| \text {. }
$$

Take $\zeta=u-u_{h}$ in (15). A short computation shows that

$$
\|\zeta\|^{2}=\left(\zeta, L^{*} \varphi\right)=B(u ; u, \varphi)-B\left(u_{h} ; u_{h}, \varphi\right)+\left(\zeta \bar{a}_{u} \nabla \zeta-\bar{a}_{u u} \zeta^{2} \nabla u, \nabla \varphi\right),
$$

where

$$
\begin{aligned}
\bar{a}_{u}(x) & =\int_{0}^{1} a_{u}(x, u(x)-t \zeta(x)) d t \\
\bar{a}_{u u}(x) & =\int_{0}^{1}(1-t) a_{u u}(x, u(x)-t \zeta(x)) d t .
\end{aligned}
$$

With $\chi \in M_{h}$ appropriately chosen we see from (17), (6), (9), (8), Hölder's inequality, the Sobolev imbedding theorem and (4i) (in that order) that

$$
\begin{aligned}
\|\zeta\|^{2} & =B(u ; u, \varphi-\chi)-B\left(u_{h} ; u_{h}, \varphi-\chi\right)+\left(\zeta \bar{a}_{u} \nabla \zeta-\bar{a}_{u u} \zeta^{2} \nabla u, \nabla \varphi\right) \\
& =B\left(u_{h} ; \zeta, \varphi-\chi\right)+\left(\zeta \bar{a}_{u} \nabla u, \nabla(\varphi-\chi)\right)+\left(\zeta \bar{a}_{u} \nabla \zeta-\bar{a}_{u u} \zeta^{2} \nabla u, \nabla \varphi\right) \\
& \leqslant C\|\zeta\|\|\varphi-\chi\|\|+C\| \zeta\left\|_{L^{3}(\Omega)}\right\| \zeta\|\| \varphi \|_{w_{6}^{1}(\Omega)} \\
& \leqslant C\|\zeta\|\left[h+\|\zeta\|_{L^{3}(\Omega)}\right]\|\varphi\|_{2} .
\end{aligned}
$$

Thus (16) and (13) imply that

$$
\|\zeta\| \leqslant C\|\zeta \mid\|\left[h+\|\zeta\|_{L^{3}(\Omega)}\right] \leqslant C\left[\|\zeta\| h+\|\zeta\|^{3 / 2}\|\zeta\|^{1 / 2}\right] .
$$

Hence,

$$
\|\zeta\| \leqslant C\left[\|\zeta\| h+\|\zeta\|^{3}\right]
$$

Using (14) in (18), we see that for $h$ sufficiently small

$$
\|\zeta\|+h\|\xi\| \leqslant C\left[h^{s}+\|\xi\|^{3}\right] \text {. }
$$


The inequality (19) proves the theorem provided we can show that $\|\zeta\| \rightarrow 0$ as $h \rightarrow 0$; this is done via a compactness argument. From (12) and the fact that $|a(x, z)|$ $\leqslant \alpha_{1}$ for $(x, z) \in \bar{\Omega} \times \mathbf{R}$ we see that there is a constant independent of $h$ such that $\left\|u_{h}-u\right\| \leqslant C$. Hence

$$
\left|u_{h}-g\right| \leqslant C h^{1 / 2}
$$

and

$$
\left\|u_{h}\right\|_{1} \leqslant C+\|u\|_{1} .
$$

From (21) we see that from any sequence of $h$ 's tending to zero we can choose a subsequence $\left\{h_{k}\right\}_{k=1}^{\infty}$ such that for some $w \in W_{2}^{1}(\Omega), u_{h_{k}} \rightarrow w$ in $L^{2}(\Omega)$ and weakly in $W_{2}^{1}(\Omega)$. It follows from $(20)$ and the trace inequality

that

$$
|\varphi| \leqslant C\|\varphi\|^{1 / 2}\|\varphi\|_{1}^{1 / 2}, \quad \varphi \in W_{2}^{1}(\Omega),
$$

$$
w=g \quad \text { on } \partial \Omega .
$$

We want to show that $w=u$ by showing that $w$ is a weak solution of (1). Take $v \in$ $C_{0}^{\infty}(\Omega)$ and $v_{h_{k}} \in M_{h_{k}}$ such that $v_{h_{k}}$ has compact support in $\Omega$ and

$$
\left\|v-v_{h_{k}}\right\|_{1}=o(1) \quad \text { as } k \rightarrow \infty \text {. }
$$

Then

$$
\begin{aligned}
|(a(w) \nabla w, \nabla v)-(f, v)|= & \mid\left(a(w) \nabla w, \nabla\left(v-v_{h_{k}}\right)\right) \\
& +\left(a(w) \nabla w-a\left(u_{n_{k}}\right) \nabla u_{h_{k}}, \nabla v_{h_{k}}\right)-\left(f,\left(v-v_{h_{k}}\right)\right) \mid \\
\leqslant & C\left\|v-v_{h_{k}}\right\|_{1}+\left|\left(a(w) \nabla w-a\left(u_{h_{k}}\right) \nabla u_{h_{k}}, \nabla v\right)\right| .
\end{aligned}
$$

Rewriting the last term as

$$
\left(a(w) \nabla\left(w-u_{h_{k}}\right), \nabla v\right)+\left(\left(a(w)-a\left(u_{h_{k}}\right)\right) \nabla u_{h}, \nabla v\right)
$$

and using the convergence of $u_{h_{k}}$ to $w$, we see that

$$
(a(w) \nabla w, \nabla v)=(f, v), \quad v \in C_{0}^{\infty}(\Omega) .
$$

Thus we see from (22) and (23) that $w$ is a weak solution in $W_{2}^{1}(\Omega)$ of (1). Assume for the moment that such weak solutions are unique. Then $w=u$, and it follows by a standard argument that $u_{h} \rightarrow u$ in $L^{2}(\Omega)$ as $h \rightarrow 0$. Hence, $\|\zeta\| \rightarrow 0$ as $h \rightarrow 0$, and the conclusion (11) follows from (19).

To show that weak solutions of (1) are unique we shall first use a "boot-strap" argument to see that weak solutions are smooth solutions and then apply the results of [1]. Suppose that $w \in W_{2}^{1}(\Omega)$ satisfies (22) and (23). Then by results of de Giorgi and Nash (see [2, Theorem 5.3.7]) $w$ is in fact Hölder continuous on $\bar{\Omega}$. (We always choose the smooth element of the equivalence class in $W_{2}^{1}(\Omega)$ if there is one.) It then follows from a result of Morrey (see [2, Theorem 5.5.4]) that $w \in W_{p}^{1}(\Omega)$ for $1<p<$ $\infty$; in particular, $w \in W_{4}^{1}(\Omega)$. This then implies that $w \in W_{2}^{2}(\Omega)$, since $w$ is a weak solution of 


$$
\begin{aligned}
-\Delta \varphi & =(1 / a)(\nabla a(\varphi)) \cdot \nabla \varphi+f \text { on } \Omega, \\
\varphi & =g \text { on } \partial \Omega .
\end{aligned}
$$

Finally we can apply the Schauder estimates (see [2, Theorem 5.6.3]) to see that $w \in$ $C^{2+\alpha}(\bar{\Omega})$. Thus, weak solutions of (1) are in fact classical solutions of (1) and belong to $C^{2}(\bar{\Omega})$; therefore, Theorem 1 of [1] implies that these solutions are unique. This completes the proof of Theorem 1 .

4. Newton's Method. Using an additional hypothesis on the function spaces $M_{h}$, we shall find conditions under which Newton's method can be used to compute the solutions $u_{h}$ of the nonlinear algebraic equations (9).

For $z \in L^{2}(\Omega)$ define a bilinear form $N(z ; \cdot ;)$ on $H$ by

$$
N(z ; v, w)=B(z ; v, w)+D(z ; v, w),
$$

where $B$ is defined in (5) and

$$
D(z ; v, w)=\left(a_{u}(z) v \nabla z, \nabla w\right) .
$$

Given $z_{0} \in M_{h}$, the Newton approximations to $u_{h}$ form a sequence $\left\{z_{k}\right\}_{k=0}^{\infty}$ in $M_{h}$ satisfying

$$
N\left(z_{k} ; z_{k+1}-z_{k}, v\right)=(f, v)-\left\langle g, a(g) \frac{\partial v}{\partial v}-\gamma h^{-1} v\right\rangle-B\left(z_{k} ; z_{k}, v\right), \quad v \in M_{h}
$$

For each $M_{h}$ let

$$
\sigma_{h}=\sup \left\{\|v\|_{L^{\infty}(\Omega)} /\|v\|_{1}: \quad 0 \neq v \in M_{h}\right\}
$$

We shall assume throughout this section that $u_{h}$ converges to $u$ sufficiently rapidly that

$$
\left\|u-u_{h}\right\|_{L^{\infty}(\Omega)}+\sigma_{h}\left\|u-u_{h}\right\|_{1} \rightarrow 0 \text { as } h \rightarrow 0 .
$$

In addition, we shall assume that there is a constant $c_{4}$ independent of $h$, such that

$$
\left\|u_{h}\right\|_{w_{\infty}^{1}(\Omega)} \leqslant c_{4}
$$

in many cases this can be verified by using Theorem 1 together with approximation and inverse assumptions which are slightly stronger than those made in (4).

Our main result for Newton's method is the following.

THEOREM 2. There exist positive constants $h_{0}, \delta$ and $c_{5}$ such that, if $0<h \leqslant$ $h_{0}$ and $\sigma_{h}\left\|z_{0}-u_{h}\right\|_{1} \leqslant \delta$, then $\left\{z_{k}\right\}_{k=0}^{\infty}$ exists and $\nu_{k}=\left\|z_{k}-u_{h}\right\|$ is a decreasing sequence satisfying

$$
v_{k+1} \leqslant c_{5} \sigma_{h}\left\|z_{k}-u_{h}\right\|_{1}^{2} \leqslant c_{5} \sigma_{h} \nu_{k}^{2} .
$$

The proof of Theorem 2 relies heavily on the following lemma.

LEMMA 2. Given $\tau>0$, there exist positive constants $\delta, h_{0}$ and $c_{6}$ such that the following holds. If $0<h \leqslant h_{0}$, if $z \in W_{\infty}^{1}(\Omega)$ satisfies

$$
\|z\|_{W_{\infty}^{1}(\Omega)} \leqslant \tau \text { and }\|z-u\|_{1} \sigma_{h} \leqslant \delta,
$$

and if $G$ is a linear functional on $H$ with norm $\|G\|$, then there exists a unique $v \in M_{h}$ satisfying the equations 


$$
N(z ; v, w)=G(w), \quad w \in M_{h} .
$$

Furthermore, $v$ satisfies the bound

$$
\|v\|\left\|\leqslant c_{6}\right\| G \| .
$$

Proof. It suffices to show that (32) holds, since it implies that solutions of (31) are unique and hence exist.

From Lemma 1 it follows that

Hence,

$$
\begin{aligned}
\rho\|v\| \|^{2} & \leqslant B(z ; v, v)=G(v)-D(z ; v, v) \\
& \leqslant\left[\|G\|+\left\|a_{u}(z) \nabla z\right\|_{L^{\infty}(\Omega)}\|v\|\right]\|v\| .
\end{aligned}
$$

$$
\|v\| \leqslant \widetilde{C}[\|G\|\|+\| v \|] .
$$

Let $L^{*}$ be as in (15), and let $\varphi$ satisfy

Then, for $\chi \in M_{h}$,

$$
L^{*} \varphi=v \quad \text { on } \Omega, \quad \varphi=0 \quad \text { on } \partial \Omega .
$$

$$
\begin{aligned}
\|v\|^{2} & =N(u ; v, \varphi) \\
& =\{N(z ; v, \varphi-\chi)+G(\varphi+(\chi-\varphi))\}+[N(u ; v, \varphi)-N(z ; v, \varphi)] .
\end{aligned}
$$

Using (4i) and the bound $\|\varphi\|_{2} \leqslant C\|v\|$, we see that

$$
\{\cdots\} \leqslant C(h\|v\|\|+\| G \|)\|v\| .
$$

A short computation shows that

$$
\begin{aligned}
{[\cdots] } & \leqslant C\|u-z\|_{L^{\infty}(\Omega)}\|v\|_{1}\|\varphi\|_{1}+C\|v\|_{L^{\infty}(\Omega)}\|u-z\|\left\|_{1}\right\| \varphi \|_{1} \\
& \leqslant C\left(\left\|u-u_{h}\right\|_{L^{\infty}(\Omega)}+\sigma_{h}\left(\left\|u-u_{h}\right\|_{1}+\left\|u_{h}-z\right\|_{1}\right)\right)\|v\|_{1}\|v\| .
\end{aligned}
$$

Thus (34), (35) and (36) imply that

$$
\|v\| \leqslant C\|G\|+F\|\| v \|,
$$

where

$$
F=C\left(h+\left\|u-u_{h}\right\|_{L^{\infty}(\Omega)}+\sigma_{h}\left(\left\|u-u_{h}\right\|_{1}+\left\|u_{h}-z\right\|_{1}\right)\right) .
$$

From (33) and (37) it follows that

$$
(-1-\tilde{C} F)\|v\|\|\leqslant C\| G \| .
$$

If $h_{0}$ and $\delta$ are taken sufficiently small that $\widetilde{C} F \leqslant 1 / 2$, then (38) implies (32). This completes the proof of the lemma.

Proof of Theorem 2. First we show that, if $h_{0}$ and $\delta$ are sufficiently small, if $0<h \leqslant h_{0}$, and if $\sigma_{h}\left\|z_{k}-u_{h}\right\| \leqslant \delta$, then there exists a unique $z_{k+1}$. It suffices to show that, if $v \in M_{h}$ satisfies

$$
N\left(z_{k} ; v, w\right)=0, \quad w \in M_{h},
$$

then $v \equiv 0$. To see this, note that

$$
N\left(u_{h} ; v, w\right)=G(w), \quad w \in M_{h},
$$


where

$$
G(w)=\left(\left[a\left(u_{h}\right)-a\left(z_{k}\right)\right] \nabla v, \nabla w\right)+\left(\left[\left(a\left(u_{h}\right)-a\left(z_{k}\right)\right) \nabla u_{h}-a\left(z_{k}\right) \nabla\left(z_{k}-u_{h}\right)\right] v, \nabla w\right) .
$$

It is easily checked that $\|G\| \leqslant C \sigma_{h}\left\|u_{h}-z_{k}\right\|_{1}\|v\|_{1}$. Hence, by Lemma 2 , $\|v\| \| \leqslant$ $c_{6} C \sigma_{h}\left\|u_{h}-z_{k}\right\|_{1}\|v\| \|$; thus, $v \equiv 0$ if $c_{6} C \delta<1$.

To verify $(*)$, note that

$$
\begin{aligned}
N\left(u_{h} ; z_{k+1}-u_{h}, w\right)= & N\left(u_{h} ; z_{k}-u_{h}, w\right)+N\left(z_{k} ; z_{k+1}-z_{k}, w\right) \\
& +\left[N\left(u_{h} ; z_{k+1}-z_{k}, w\right)-N\left(z_{k} ; z_{k+1}-z_{k}, w\right)\right] \\
= & \left\{N\left(u_{h} ; z_{k}-u_{h}, w\right)+B\left(u_{h} ; u_{h}, w\right)-B\left(z_{k} ; z_{k}, w\right)\right\} \\
& +\left[N\left(u_{h} ; z_{k+1}-z_{k}, w\right)-N\left(z_{k} ; z_{k+1}-z_{k}, w\right)\right] \\
= & G_{1}(w)+G_{2}(w)=G(w), \quad w \in M_{h},
\end{aligned}
$$

where the braces and brackets indicate the decomposition of $G$ into $G_{1}$ and $G_{2}$. Since

$$
G_{1}(w)=\left(1 / \tilde{a}_{u u}\left(z_{k}-u_{h}\right)^{2} \nabla u_{h}+\tilde{a}_{u}\left(z_{k}-u_{h}\right) \nabla\left(u_{h}-z_{k}\right), \nabla w\right),
$$

where $\tilde{a}_{u u}$ and $\tilde{a}_{u}$ are certain averages of $a_{u u}$ and $a_{u}$, respectively, it follows that

$$
\left\|G_{1}\right\| \leqslant C \sigma_{h}\left\|z_{k}-u_{h}\right\|_{1}^{2} \leqslant C \sigma_{h} \nu_{k}^{2} .
$$

Similarly, since

$$
\begin{aligned}
G_{2}(w)= & \left(\left[a\left(u_{h}\right)-a\left(z_{k}\right)\right] \nabla\left(z_{k+1}-z_{k}\right), \nabla w\right) \\
& +\left(\left[\left(a_{u}\left(u_{h}\right)-a_{u}\left(z_{k}\right)\right) \nabla u_{h}+a_{u}\left(z_{k}\right) \nabla\left(u_{h}-z_{k}\right)\right]\left(z_{k+1}-z_{k}\right), \nabla w\right),
\end{aligned}
$$

we see that

$$
\begin{aligned}
\left\|G_{2}\right\| & \leqslant C \sigma_{h}\left\|z_{k}-u_{h}\right\|_{1}\left(\left\|z_{k}-u_{h}\right\|_{1}+\left\|z_{k+1}-u_{h}\right\|_{1}\right) \\
& \leqslant C \sigma_{h} \nu_{k}\left(\nu_{k}+\nu_{k+1}\right) .
\end{aligned}
$$

The conclusion, (*), then follows from Lemma 2, (39), (40), and (41).

In the cases where $M_{h}$ is obtained from piecewise polynomial functions over a quasi-regular triangulation of $\Omega$, one can show that

$$
\sigma_{h} \approx \ln h^{-1}, \quad n=2, \quad \text { or } \sigma_{h} \approx h^{-1 / 2}, \quad n=3 .
$$

Department of Mathematics

University of Chicago

Chicago, Illinois 60637

1. J. DOUGLAS, JR., T. DUPONT \& J. SERRIN, "Uniqueness and comparison theorems for nonlinear elliptic equations in divergence form," Arch. Rational Mech. Anal., v. 42, 1971, pp. 157-168.

2. C. B. MORREY, JR., Multiple Integrals in the Calculus of Variations, Die Grundlehren der math. Wissenschaften, Band 130, Springer-Verlag, New York, 1966. MR 34 \#2380.

3. J. A. NITSCHE, “Über ein Variationsprinzip zur Lösung von Dirichlet-Problemen bei Verwendung von Teilräumen, die kleinen Randbedingungen unterworfen sind," Abh. Math. Sem. Univ. Hamburg, v. 36, 1970/71, pp. 9-15. 